\title{
Molecular Subtyping and Tracking of Listeria monocytogenes in Latin-Style Fresh-Cheese Processing Plants
}

\author{
D. Y. Kabuki, ${ }^{\star}$ A. Y. Kuaye, ${ }^{*}$ M. Wiedmann, and K. J. Boor \\ Department of Food Science, Cornell University, Ithaca, NY 14853
}

\section{ABSTRACT}

Latin-style fresh cheeses, which have been linked to at least 2 human listeriosis outbreaks in the United States, are considered to be high-risk foods for Listeria monocytogenes contamination. We evaluated L. monocytogenes contamination patterns in 3 Latin-style freshcheese processing plants to gain a better understanding of $L$. monocytogenes contamination sources in the manufacture of these cheeses. Over a 6-mo period, 246 environmental samples were collected and analyzed for $L$. monocytogenes using both the Food and Drug Administration (FDA) method and the Biosynth L. monocytogenes detection system (LMDS). Finished cheese samples from the same plants $(\mathrm{n}=111)$ were also analyzed by the FDA method, which was modified to include $L$. monocytogenes plating medium (LMPM) and the $L$. monocytogenes confirmatory plating medium (LMCM) used in the LMDS method. Listeria monocytogenes was detected in $6.3 \%$ of cheese and $11.0 \%$ of environmental samples. Crates, drains, and floor samples showed the highest contamination rates, with 55.6, 30.0, and $20.6 \%$ L. monocytogenes positive samples, respectively. Finished products and food contact surfaces were positive in only one plant. The FDA method showed a higher sensitivity than the LMDS method for detection of $L$. monocytogenes from environmental samples. The addition of LMPM and LMCM media did not further enhance the performance of the FDA method for L. monocytogenes detection from finished products. Molecular subtyping (PCR-based allelic analysis of the virulence genes act $A$ and $h l y$ and automated ribotyping) was used to track contamination patterns. Ribotype DUP-1044A, which had previously been linked to a 1998 multistate human listeriosis outbreak in the United States, was the most commonly identified subtype (20/36 isolates) and was isolated from 2 plants. This ribotype was persistent and widespread in one factory, where it was also responsible for the contamination of finished products.

Received January 14, 2004.

Accepted April 15, 2004.

Corresponding author: K. J. Boor; e-mail: kjb4@cornell.edu.

*Current address: Faculdade de Engenharia de Alimentos, Universidade Estadual de Campinas, Campinas - SP, Brazil.
We hypothesize that this ribotype may represent a clonal group with a specific ability to persist in food processing environments. While previous listeriosis outbreaks were linked to Latin-style fresh cheeses made from unpasteurized milk, the presence of this organism in pasteurized cheese products illustrates that persistent environmental contamination also represents an important source of finished product contamination.

(Key words: Listeria monocytogenes, Latin-style fresh cheese, molecular subtyping)

Abbreviation key: BCM = biosynth chromogenic medium, BHI = brain heart infusion, $\mathbf{C D C}=$ Centers for Disease Control and Prevention, EB = enrichment broth, LMCM = Listeria monocytogenes confirmation media, LMDS $=L$. monocytogenes detection system, LMPEB $=L$. monocytogenes pre-enrichment broth, LMPM $=L$. monocytogenes plating medium, PBBA = purple broth base agar, PFGE = pulsed-field gel electrophoresis, PI-PLC = phosphatidylinositol phospholipase C, RAPD = random amplification of polymorphic DNA, RTE = ready-to-eat.

\section{INTRODUCTION}

Listeria monocytogenes is a foodborne pathogen that causes serious invasive disease in humans. The Centers for Disease Control and Prevention (CDC) estimate that $L$. monocytogenes causes about 2500 cases and 500 deaths annually in the United States (Mead et al., 1999). A variety of different food products, including dairy products, have been implicated in human listeriosis cases and outbreaks (Fleming et al., 1985; Azadian et al., 1989; Bille, 1990; McLauchlin et al., 1990; Farber and Peterkin, 1991; Dalton et al., 1997). In particular, Latin-style fresh cheeses were responsible for a large human listeriosis outbreak in California in 1985 (Linnan et al., 1988) and more recently, for an outbreak in North Carolina (Anonymous, 2001). Beyond these outbreaks, L. monocytogenes also has been isolated from Latin-style fresh cheeses from many different countries (Destro et al., 1991; Genigeorgis et al., 1991; El Marrakchi et al., 1993; Silva et al., 1998; Saltijeral et al., 1999). The term, "Latin-style fresh cheese" represents a heter- 
ogenous group of white, unripened soft cheeses, typically bearing between 1.0 to $3.0 \%$ salt (Torres and Chandan, 1981). The Latin-style cheese industry is a rapidly expanding segment of the US dairy industry; production of these products increased from 67 million lbs. in 1996 to 96 million lbs. in 2000 (USDA-NASS, 2002). This sector of the cheese industry is largely comprised of small- and medium-sized operations. For many of these operations, the owners and employees have had little previous commercial food processing experience. Control of $L$. monocytogenes in the manufacture of Latin-style fresh cheeses represents a critical need in the US dairy industry.

Product contamination with $L$. monocytogenes results in serious economic consequences for food processors, including costly product recalls and lawsuits. Dairy products represented the food category most frequently associated with recalls due to microbial contamination reported to the FDA during fiscal years 1994 to 1998 (Wong et al., 2000). During this period, dairy product recalls accounted for $304(22 \%)$ of all food recalls, with L. monocytogenes isolated from $64.5 \%$ of these products. Since pasteurization efficiently kills $L$. monocytogenes, contamination of finished dairy products, as well as of other ready-to-eat (RTE) food products, appears most commonly to originate from postprocessing environmental sources. As L. monocytogenes is regularly isolated from food and dairy processing environments (Cox et al., 1989; Klausner and Donnelly, 1991; Jacquet et al., 1993; Pritchard et al., 1995; Sutherland and Porritt, 1996), a better understanding of the spread and survival of $L$. monocytogenes in food processing environments and of specific contributions of environmental sources to finished product contamination are needed to develop improved Listeria control strategies.

Listeria monocytogenes isolation and subtyping represent 2 critical steps in characterizing environmental contamination patterns. Classical culture-based methods for isolating $L$. monocytogenes are time consuming, typically requiring 5 to $7 \mathrm{~d}$. Recently, differential media based on detection of phosphatidylinositol phospholipase C enzyme (PI-PLC) activity have been described and evaluated for isolation of $L$. monocytogenes from food and environmental samples (Restaino et al., 1999; Karpsková et al., 2000; Hoffman and Wiedmann, 2001). The PI-PLC is an enzyme that is only produced by L. monocytogenes and Listeria ivanovii. Listeria ivanovii is uncommon in foods and food-processing environments (Arimi et al., 1997) and can easily be differentiated from $L$. monocytogenes using biochemical tests. The Biosynth L. monocytogenes detection system (LMDS) contains fluorogenic and chromogenic PI-PLC substrates in both the selective enrichment and plating media, which can enable more rapid screening and dif- ferentiation of $L$. monocytogenes. The Biosynth-LMDS method should thus allow for more rapid and sensitive detection of L. monocytogenes (Restaino et al., 1999) as compared with the FDA method, which includes the selective media Oxford and PALCAM, which are not differential for L. monocytogenes. Molecular typing methods, such as pulsed-field gel electrophoresis (PFGE), random amplification of polymorphic DNA (RAPD) and ribotyping, provide appropriate tools for tracking bacterial contamination sources throughout food systems (Autio et al., 1999; Johansson et al., 1999; Miettinen et al., 1999; Norton et al., 2001; Hoffman et al., 2003) and also are increasingly used by public health laboratories to detect and track human listeriosis outbreaks (Jacquet et al., 1995; Dalton et al., 1997).

The major goal of this research was to explore the use of different detection strategies and molecular subtyping methods to characterize $L$. monocytogenes contamination patterns in Latin-style fresh-cheese processing plants. Our results show that persistent environmental contamination in the processing plant represents a major source of finished product contamination. The persistent presence of $L$. monocytogenes in these operations demonstrates the importance of designing and implementing effective strategies for eliminating environmental $L$. monocytogenes strains in Latin-style fresh cheese and other dairy-processing plants.

\section{MATERIALS AND METHODS}

\section{Processing Plant and Sample Collection}

A total of 357 samples were collected from 3 Latinstyle fresh-cheese processing plants located in New York City during 4 visits to each facility over a 6-mo period (June to December 2000). All 3 plants purchase pasteurized milk for cheese manufacture. Cheeses are manufactured by heat treating the pasteurized milk, adding rennet and starter culture and/or acidulant (e.g., lactic or acetic acid) to obtain a curd, draining the whey, pressing the curd, followed by milling, salting, molding, and packaging. The plants were selected to represent small to relatively large-scale manufacturers (up to 18,000 gallons of pasteurized milk per day). Plant A is a large manufacturer, processing between 12,000 to 18,000 gallons per day, plant B manufactures 75 gallons twice a week in a very small processing area, and plant C, a medium-size factory, processes 1200 gallons per day.

Samples collected included 111 finished products (fresh cheeses) and 246 environmental samples (119 food contact surfaces, 40 floor drains, 34 floors, 38 walls, and 15 miscellaneous sampling sites). Food contact surfaces tested included equipment surfaces (coagulation 
vat, pressing machine, pasteurizer valve, grinder, mixer), stirring tools, a stainless steel container, tables (stainless steel and polytetrafluorethylene), a plastic strainer, a plastic barrel, padding, a plastic connecting tube (used to connect a milk stainless steel pipeline from the holding tank to the pasteurizer and from the pasteurizer to the coagulation vat). Whenever possible, environmental samples were collected from the same sites on each visit. Environmental samples were collected using a commercial environmental sponge sampling kit (Bacti Sponge Kit, Hardy Diagnostics, Santa Maria, CA) and a neutralizing buffer (Hardy Diagnostics) to neutralize the chlorine-based sanitizers and quaternary ammonium compounds used in the plants. Cheeses were collected as finished packaged products. All samples were kept refrigerated and were analyzed within $48 \mathrm{~h}$.

\section{Listeria monocytogenes Detection}

Finished products were analyzed by the FDA method (Hitchins, 1998), which was modified to also include plating of the enrichments on L. monocytogenes plating medium (LMPM, Biosynth Biochemica \& Synthetica [BCM], Naperville, IL) and L. monocytogenes confirmation media (LMCM, Biosynth), as described by Restaino et al. (1999). Briefly, 25-g samples of cheese were aseptically added to $225 \mathrm{~mL}$ of enrichment broth (EB) (Hitchins, 1998) and homogenized in a Stomacher (Seward Ltd., London, UK). The homogenized samples were incubated at $30^{\circ} \mathrm{C}$, and selective agents were added after $4 \mathrm{~h}$ of incubation, as described by Hitchins (1998). After 24 and $48 \mathrm{~h}$ of incubation, aliquots from EB were streaked onto Oxford (Difco Laboratories, Detroit, MI), PALCAM (Oxoid Ltd., Basingstoke, Hampshire, England), and LMPM. Typical Listeria-like colonies from Oxford and PALCAM were purified on brain heart infusion agar (BHI, Difco) and screened by PCR, as described below, to identify L. monocytogenes isolates. Typical PI-PLC positive Listeria-like colonies from LMPM were streaked onto LMCM and onto purple broth base agar (PBBA, Difco) containing 1.0\% rhamnose and scored according to the manufacturer's directions and as previously described (Restaino et al., 1999). Isolates confirmed as $L$. monocytogenes were verified as L. monocytogenes using the PCR assay described below.

Environmental sponge samples were analyzed using both the FDA method (Hitchins, 1998) and the L. monocytogenes detection system (LMDS, Biosynth) (Restaino et al., 1999). The sponges were homogenized manually in their bags and aseptically cut in half; one half was added to $50 \mathrm{~mL}$ of EB and the second half was added to $50 \mathrm{~mL}$ of $L$. monocytogenes preenrichment broth (LMPEB, Biosynth). The sponges were homoge- nized for $30 \mathrm{~s}$ in a Stomacher blender and analyzed following the previously published protocols for the FDA method (Hitchins, 1998) and LMDS (Restaino et al., 1999).

\section{PCR-Based Identification of Listeria monocytogenes}

Four Listeria-suspect colonies from each selective agar (Oxford, PALCAM, and/or LMCM) were screened using a $L$. monocytogenes specific PCR assay targeting the L. monocytogenes listeriolysin $\mathrm{O}$ gene, $h l y$, as previously described (Norton et al., 2000).

\section{Virulence Gene Allele Characterization}

One L. monocytogenes isolate from each selective plating medium was selected for further characterization. Thus, for samples that yielded positive results on all 3 selective media (Oxford, PALCAM, and/or LMCM), 3 isolates were further characterized. Virulence gene allele typing of actA and $h l y$ by PCR-RFLP was performed as previously described (Wiedmann et al., 1997). Briefly, hly was characterized by PCR- RFLP using the restriction endonucleases HhaI and HpaII. One of 8 possible allelic types was assigned based on the resulting restriction pattern. Following amplification of act $A$, one of 2 possible allelic types (either type "3" or "4") was assigned based on a PCR product size difference, which corresponds with the presence or absence of a 105 nucleotide region encoding a proline-rich repeat structure (Wiedmann et al., 1997).

\section{Ribotyping}

For each sample positive for $L$. monocytogenes, one isolate of each different act A and hly polymorphic type was selected for ribotype analysis. Ribotyping was performed using the restriction endonuclease EcoRI and the RiboPrinter Microbial Characterization System (Qualicon, Inc., Wilmington, DE) as described previously (Bruce, 1996). Images were acquired with a charge-coupled device camera and processed using the Riboprinter's custom software. This software normalizes fragment pattern data for band intensity and relative band position (Bruce, 1996). Ribotype patterns were automatically assigned a DuPont ID (e.g., DUP1039) by the Riboprinter, which was confirmed by visual inspection. If visual inspection found a given DuPont ID included more than one distinct ribotype pattern, which generally differed by position of only a single weak band, then each pattern was designated with an additional alphabetized letter (e.g., DUP-1039A and DUP-1039B). 
Table 1. Occurrence of Listeria monocytogenes in Latin-style fresh-cheese processing plants.

\begin{tabular}{|c|c|c|c|c|c|c|}
\hline \multirow[b]{2}{*}{ Plant } & \multirow[b]{2}{*}{ Visit } & \multicolumn{2}{|c|}{ Finished products ${ }^{1}$} & \multicolumn{2}{|c|}{ Environmental samples } & \multirow[b]{2}{*}{$\begin{array}{l}\text { No. of } \\
\text { Listeria spp. } \\
\text { positive samples }\end{array}$} \\
\hline & & $\begin{array}{l}\text { No. of } \\
\text { samples }\end{array}$ & $\begin{array}{l}\text { No. of Listeria } \\
\text { monocytogenes } \\
\text { positive samples }\end{array}$ & $\begin{array}{l}\text { No. of } \\
\text { samples }\end{array}$ & $\begin{array}{l}\text { No. of } L . \\
\text { monocytogenes } \\
\text { positive samples }\end{array}$ & \\
\hline \multirow[t]{5}{*}{ A } & 1 & 10 & 0 & 27 & 2 & 5 \\
\hline & 2 & 18 & 0 & 23 & 1 & 5 \\
\hline & 3 & 18 & 0 & 23 & 1 & 3 \\
\hline & 4 & 18 & 0 & 24 & 0 & 5 \\
\hline & Subtotal & 64 & $0(0.0 \%)$ & 97 & $4(4.1 \%)$ & $18(18.5 \%)$ \\
\hline \multirow[t]{5}{*}{ B } & 1 & 5 & 0 & 17 & 3 & 1 \\
\hline & 2 & 6 & 0 & 13 & 2 & 0 \\
\hline & 3 & 6 & 0 & 13 & 1 & 2 \\
\hline & 4 & 6 & 0 & 12 & 2 & 0 \\
\hline & Subtotal & 23 & $0(0.0 \%)$ & 55 & $8(14.5 \%)$ & $3(5.4 \%)$ \\
\hline \multirow[t]{6}{*}{$\mathrm{C}$} & 1 & 6 & 1 & 23 & 5 & 3 \\
\hline & 2 & 6 & 3 & 22 & 6 & 1 \\
\hline & 3 & 6 & 3 & 25 & 2 & 1 \\
\hline & 4 & 6 & 0 & 24 & 2 & 1 \\
\hline & Subtotal & 24 & $7(29.2 \%)$ & 94 & $15(15.9 \%)$ & $6(6.4 \%)$ \\
\hline & Total & 111 & $7(6.3 \%)$ & 246 & $27(11.0 \%)$ & $27(11.0 \%)$ \\
\hline
\end{tabular}

${ }^{1}$ If finished product samples were negative for L. monocytogenes, they were also negative for Listeria spp.

${ }^{2}$ Number of samples negative for L. monocytogenes, but positive for Listeria spp.

\section{RESULTS}

\section{Occurrence of Listeria spp. and L. monocytogenes in Cheese and Environmental Samples}

Environmental and cheese samples were collected from 3 processing plants over a total of 4 visits to each plant. Listeria monocytogenes was detected in $6.3 \%$ of finished product samples and in $11.0 \%$ of environmental samples (Table 1). Only finished products from one plant (plant C) were positive for L. monocytogenes. Environmental samples from plant A showed a lower frequency of $L$. monocytogenes contamination $(4.1 \%)$ as compared with plants B and C, which showed contamination frequencies of 14.5 and $15.9 \%$, respectively (Table 1). All 3 plants showed similar prevalences of Listeria spp. in environmental samples (20.0 to 22.7\%). Among the environmental samples, L. monocytogenes was most commonly isolated from drains (30.0\%) and floors (20.6\%) (Table 2). Only 2 food contact surface

Table 2. Occurrence of Listeria monocytogenes in environmental samples collected in 3 Latin-style soft cheese processing plants.

\begin{tabular}{lcc}
$\begin{array}{l}\text { Type of surface } \\
\text { sampled }\end{array}$ & $\begin{array}{l}\text { No. of samples } \\
\text { tested }\end{array}$ & $\begin{array}{l}\text { No. of positive } \\
\text { samples }(\%)\end{array}$ \\
\hline Food contact surfaces & 119 & $2^{1}(1.7 \%)$ \\
Floor drains & 40 & $12(30.0 \%)$ \\
Floors & 34 & $7(20.6 \%)$ \\
Walls & 38 & 0 \\
Miscellaneous & 15 & $6^{2}(40.0 \%)$ \\
TOTAL & 246 & $27(11.0 \%)$ \\
\hline
\end{tabular}

\footnotetext{
${ }^{1}$ Positive samples were a plastic connecting tube and a polytetrafluorethylene table (plant C).

${ }^{2}$ Positive samples were crates and plastic milk packages.
}

samples (a plastic connecting tube at the exit of the pasteurizer, which was used to transfer milk to the coagulation vat, and a polytetrafluorethylene table; Table 2), both collected in plant C, were positive for $L$. monocytogenes. Crates were contaminated with $L$. monocytogenes in all 3 plants (Table 3 ).

\section{Molecular Subtyping}

A total of $80 \mathrm{~L}$. monocytogenes, representing up to 3 isolates from each positive sample (one isolate from each selective plating media), were initially subtyped using allelic analysis of the virulence genes act $\mathrm{A}$ and $h l y$. Both act A types 3 and 4 were identified among the isolates. Although 8 different $h l y \mathrm{~A}$ types exist (Wiedmann et al., 1997), only types 1 and 2 were identified among the isolates collected in this study. With 2 exceptions, multiple isolates from the same site always displayed the same $h l y$ and $a c t A$ types. Specifically, sponge samples collected from crates in plant B at visits 2 and 4 each contained 2 different actA and hly subtypes (Table 3). Based on these initial subtyping results, 36 representative isolates (one from each positive sample, unless multiple subtypes were observed based on hly and act $A$ allelic analysis) were selected for further subtyping by automated EcoRI ribotyping. Ribotyping discriminated 8 distinct patterns among the 36 isolates (Table 3). Ribotype DUP-1044A/hly type 1, actA type 4 represented the most commonly isolated subtype (55.6\%). Two ribotypes (DUP-1044A and DUP-1062C) were identified among the isolates from plant A, whereas 5 ribotypes (DUP-1042A, DUP-1042C, DUP1049A, DUP-1062C, and DUP-1052) and 3 ribotypes 
Table 3. Listeria monocytogenes contamination patterns and subtyping results for 3 Latin-style soft cheese processing plants.

\begin{tabular}{|c|c|c|c|c|c|c|c|}
\hline Factory & Visit & Sites (sample code) & $\begin{array}{l}\text { act A } \\
\text { type }\end{array}$ & $\begin{array}{l}\text { hlyA } \\
\text { type }\end{array}$ & Lineage & $\begin{array}{l}\text { Ribotype } \\
\text { (DUP) }\end{array}$ & RiboPrinter pattern \\
\hline \multirow[t]{4}{*}{$\mathbf{A}$} & 1 & Drain 10 , processing area 2 & 4 & 1 & I & $1044 \mathrm{~A}$ & $1 \mathrm{ll}$ \\
\hline & & Floor, processing area 2 & 4 & 1 & $\mathbf{I}$ & $1044 \mathrm{~A}$ & \\
\hline & 2 & Drain, cooler room & 4 & 2 & II & $1062 \mathrm{C}$ & $x_{1}$ \\
\hline & 3 & Crate & 4 & 2 & II & $1062 \mathrm{C}$ & 1 \\
\hline \multirow[t]{10}{*}{ B } & 1 & Drain, processing area & 4 & 1 & $\mathbf{I}$ & $1042 \mathrm{C}$ & 1 \\
\hline & & Floor, processing area & 4 & 1 & $\mathbf{I}$ & $1042 \mathrm{C}$ & 1 \\
\hline & & Crate & 3 & 1 & I & $1042 \mathrm{~A}$ & 1 \\
\hline & 2 & Drain, processing area & 4 & 1 & $\mathbf{I}$ & $1042 \mathrm{C}$ & \\
\hline & & Crate $^{1}$ & 3 & 1 & I & $1042 \mathrm{~A}$ & | \\
\hline & & & 4 & 2 & II & 1049A & \\
\hline & 3 & Drain, processing area & 4 & 1 & I & $1042 C$ & 1 \\
\hline & 4 & Crate $^{1}$ & 3 & 1 & $\mathbf{I}$ & $1042 \mathrm{~A}$ & | \\
\hline & & & 4 & 2 & II & $1062 \mathrm{C}$ & 11 \\
\hline & & Milk package & 3 & 1 & $\mathbf{I}$ & 1052 & 1 \\
\hline \multirow[t]{22}{*}{$\mathbf{C}$} & 1 & Cheese & 4 & 1 & I & $1044 \mathrm{~A}$ & $\mathbf{I}$ \\
\hline & & Drain 1 , processing area 1 & 4 & 1 & $\mathbf{I}$ & $1044 \mathrm{~A}$ & \\
\hline & & Drain 2, processing area 2 & 4 & 1 & $\mathbf{I}$ & $1044 \mathrm{~A}$ & I \\
\hline & & Drain 3, processing area 3 & 4 & 1 & $\mathbf{I}$ & $1044 \mathrm{~A}$ & 1 \\
\hline & & Floor 2, processing area 2 & 3 & 2 & II & 1045B & 1 \\
\hline & & Floor 4, cooler room & 4 & 1 & $\mathbf{I}$ & $1044 \mathrm{~A}$ & \\
\hline & 2 & Cheese & 4 & 1 & $\mathbf{I}$ & $1044 \mathrm{~A}$ & 1 \\
\hline & & Cheese & 4 & 1 & $\mathbf{I}$ & $1044 \mathrm{~A}$ & 1 \\
\hline & & Cheese & 4 & 1 & I & $1044 \mathrm{~A}$ & 1 \\
\hline & & $\begin{array}{l}\text { Plastic connecting tube, } \\
\text { processing area } 2\end{array}$ & 4 & 1 & $\mathbf{I}$ & $1044 \mathrm{~A}$ & I \\
\hline & & Drain 1, processing area 2 & 4 & 1 & I & $1044 \mathrm{~A}$ & II \\
\hline & & Drain 2, processing area 2 & 3 & 2 & II & 1045B & \\
\hline & & Drain 3, processing area 3 & 4 & 1 & $\mathbf{I}$ & $1044 \mathrm{~A}$ & 1 \\
\hline & & Floor 3, processing area 3 & 4 & 1 & I & $1044 \mathrm{~A}$ & 1 \\
\hline & & Floor 4, cooler room & 4 & 1 & $\mathbf{I}$ & 1044A & 1 \\
\hline & 3 & Cheese & 4 & 1 & $\mathbf{I}$ & $1044 \mathrm{~A}$ & I \\
\hline & & Cheese & 4 & 1 & $\mathbf{I}$ & $1044 \mathrm{~A}$ & 1 \\
\hline & & Cheese & 4 & 1 & I & $1044 \mathrm{~A}$ & II \\
\hline & & Floor 4, cooler room & 4 & 1 & $\mathbf{I}$ & $1044 \mathrm{~A}$ & I \\
\hline & & Crate & 4 & 2 & II & $1039 \mathrm{C}$ & I \\
\hline & 4 & $\begin{array}{l}\text { Polytetrafluorethylene table, } \\
\text { processing area } 3\end{array}$ & 4 & 1 & $\mathbf{I}$ & $1044 \mathrm{~A}$ & I III \\
\hline & & Drain 2 , processing area 2 & 3 & 2 & II & 1045B & II \\
\hline
\end{tabular}

${ }^{1}$ Two subtypes as determined by allelic analysis of $h l y$ and actA were isolated from these samples; subtype characteristics for both strains are shown. 
Table 4. Comparison of Biosynth chromogenic medium-Listeria moncytogenes detection system (LMDS) and FDA methods for detection of $L$. monocytogenes from 246 environmental samples.

\begin{tabular}{lllllll}
\hline $\begin{array}{l}\text { Isolation } \\
\text { methods }\end{array}$ & $\begin{array}{l}\text { No. of } \\
\text { positive } \\
\text { samples }^{1}\end{array}$ & $\begin{array}{l}\text { No. of true } \\
\text { negative } \\
\text { samples }\end{array}$ & $\begin{array}{l}\text { No. of false } \\
\text { positives }\end{array}$ & $\begin{array}{l}\text { No. of false } \\
\text { negatives }\end{array}$ & $\begin{array}{l}\text { Sensitivity } \\
(\%)^{2}\end{array}$ & $\begin{array}{l}\text { Specificity } \\
(\%)^{3}\end{array}$ \\
\hline LMDS & 13 & 219 & 0 & 14 & 48.2 & 100 \\
FDA & 23 & 219 & 0 & 4 & 85.2 & 100 \\
\hline
\end{tabular}

1 The total number of positive samples identified (27) is the sum of positives detected only by LMDS plus those detected only by the FDA method, plus those detected by both.

$2 \%$ Sensitivity $=100 \times$ [true positives/ (true positives + false negatives)].

${ }^{3} \%$ Specificity $=100 \times[$ true negatives $/($ true negatives + false positives $)]$.

(DUP-1044A, DUP-1045B, and DUP-1039C) were identified in plants $\mathrm{B}$ and $\mathrm{C}$, respectively (Table 3 ).

\section{Evaluation of FDA Method and BCM-LMDS}

A total of 111 cheese samples were analyzed for $L$. monocytogenes using a modified FDA method, which included plating on Oxford, PALCAM, and LMPM. Both Oxford and PALCAM allowed L. monocytogenes isolation from 7 cheese samples, whereas LMPM allowed $L$. monocytogenes isolation from only 5 of the same samples. Some L. monocytogenes do not produce PI-PLC (Notermans et al., 1991) and, therefore, may produce false negative results on LMPM. However, isolates that had been obtained from samples that yielded positive results on Oxford and PALCAM, but negative results on LMPM, did display PI-PLC production when restreaked on LMPM.

A total of 246 environmental sponge samples were analyzed using both the FDA method and the LMDS method. Twenty-seven samples were positive for L. monocytogenes by one or both methods. The FDA method yielded 23 positive samples but failed to allow detection of $L$. monocytogenes in 4 samples that were positive by LMDS. The use of LMDS allowed isolation of $L$. monocytogenes from 13 samples, but did not detect $L$. monocytogenes in 14 samples that were positive by the FDA method (Table 4). The sensitivities of the FDA method and BCM LMDS were thus 85.2 and $48.2 \%$, respectively (Table 4). All isolates that showed reactions typical for L. monocytogenes on LMPM and LMCM were confirmed as $L$. monocytogenes by a hlyA PCR assay. For the 23 environmental samples that were positive using the FDA method, L. monocytogenes was detected in $91.3 \%$ $(21 / 23)$ of the samples after $24 \mathrm{~h}$ of enrichment; 20 and 15 samples yielded $L$. monocytogenes on PALCAM and Oxford, respectively. For 2 samples, L. monocytogenes was only isolated after $48 \mathrm{~h}$ of enrichment.

\section{DISCUSSION}

Listeria monocytogenes contamination of RTE foods appears to occur predominantly through postprocessing contamination from food-processing environments (Tompkin, 2002). Therefore, environmental testing is a critical component of $L$. monocytogenes control programs in the dairy and food-processing industries (Tompkin, 2002). Data on the efficacy of environmental testing methods and on environmental $L$. monocytogenes and Listeria spp. contamination patterns in different food processing systems are crucial for development of science-based regulations to enable better control of this food-borne pathogen at its sources. Fresh Latinstyle cheeses have been responsible for at least 2 human listeriosis outbreaks in the United States (Linnan et al., 1988; Anonymous, 2001). We selected 3 Latin-style fresh-cheese processing plants representing different scales of operation to probe environmental L. monocytogenes contamination patterns and to explore the use of different detection and subtyping methods to monitor environmental contamination patterns.

\section{L. monocytogenes and Listeria Contamination Patterns}

Listeria monocytogenes was isolated from $6.3 \%$ of finished product samples and from $11.0 \%$ of environmental samples; isolation frequencies varied considerably from plant to plant (Table 1). In 1991, Genigeorgis et al. reported a $2 \%$ L. monocytogenes prevalence in Latinstyle fresh cheeses collected in California. In 2003, Gombas et al. reported a $0.07 \%$ prevalence in cheeses tested in Northern California and a $0.28 \%$ prevalence in those tested in Maryland. L. monocytogenes prevalence in similar products tested in other countries ranged from 3.3 to $41.2 \%$ (Destro et al., 1991; El Marrakchi et al., 1993; Furlanetto et al., 1996; Silva et al., 1998; Saltijeral et al., 1999). Whereas cheeses manufactured from raw milk have been reported to bear a higher prevalence of $L$. monocytogenes than cheeses make from pasteurized milk (Genigeorgis et al., 1991; Silva et al., 1998; Saltijeral et al., 1999), our data demonstrate that L. monocytogenes can also be found in products manufactured from pasteurized milk. 
Listeria monocytogenes was found in up to $15.9 \%$ of environmental samples collected from the 3 processing plants studied; highest contamination rates were found from floor drains and floors as well as from crates. These findings are consistent with previous reports, which have also found that drain and floor samples showed the highest prevalence of $L$. monocytogenes among samples collected from dairy processing plants (Klausner and Donnelly, 1991; Pritchard et al., 1995; Sutherland and Porritt, 1996). Our observation that crates from all 3 plants were at least sporadically positive for $L$. monocytogenes also further confirms that these appliances represent an important potential source of L. monocytogenes contamination in dairy processing plants (Sutherland and Porritt, 1996). Crates had multiple possible functions within each plant, thus providing multiple opportunities for the possible spread of L. monocytogenes from point to point within a plant. To illustrate, crates were used to transport pasteurized milk packages from cooler rooms to the processing areas; to transport finished products from processing areas to cooler rooms; and to store finished products in the cooler rooms. Whereas L. monocytogenes has previously been isolated from dairy industry food contact surfaces (Jacquet et al., 1993; Pritchard et al., 1995; Sutherland and Porritt, 1996), we isolated L. monocytogenes from only 2 food contact surfaces (a connecting plastic tube coming from the pasteurizer and a polytetrafluorethylene table) in one of the 3 plants included in our study.

When comparing the 3 processing plants tested in this study, the largest plant (plant A), which had recently been constructed, showed a low incidence of $L$. monocytogenes contamination, comparable to environmental contamination prevalence data for other wellmanaged RTE food-processing plants. These data indicate that manufacturing of Latin-style fresh cheeses under appropriate conditions can produce products of comparable safety to other RTE food products. Plant B represents the smallest establishment included in this study. Whereas this plant showed a high frequency of L. monocytogenes-positive environmental samples, which are likely associated with the poor design and layout of the facility, no finished product samples were found positive for $L$. monocytogenes. In this factory, the presence of $L$. monocytogenes on the outside of packages containing incoming pasteurized milk revealed a potential route of environmental contamination. Plant $C$ was the only plant in this study that had both $L$. monocytogenes contamination of many environmental sites (Table 3) as well as contamination of finished products, most likely due to postpasteurization contamination. The plant $\mathrm{C}$ plastic food contact surfaces that were positive for $L$. monocytogenes were manufactured using materials that are difficult to sanitize (Krysinski et al.,
1992) and that appear to facilitate biofilm formation (Sinde and Carballo, 2000). These findings highlight the importance of using appropriate materials (e.g., stainless steel) and sanitary design for the manufacture of food-processing equipment.

\section{Tracking of $L$. monocytogenes Contamination Patterns by Molecular Subtyping}

Molecular subtyping (allelic analysis of actA and hly and automated ribotyping) was used to further elucidate $L$. monocytogenes contamination patterns. Initial subtyping using allelic analysis of actA and hly was used to prescreen isolates when multiple isolates from different enrichment procedures were available for a given sample. Whereas a combination of actA and hly allelic types provides less sensitive subtype differentiation as compared with more expensive methods, such as PFGE or automated ribotyping (Wiedmann, 2002), we reasoned that these methods provide a cost-effective alternative for identification of at least some samples that contain multiple L. monocytogenes subtypes. In this study, we identified 2 samples that contained multiple subtypes (Table 3). This finding is consistent with previous studies, which also described the presence of multiple subtypes within a single sample (Ryser et al., 1996). Clearly, it is possible to underestimate the true subtype diversity present in a given sample when only a single or limited number of isolates are tested from a sample. Unfortunately, cost considerations currently make it impractical to subtype large numbers of isolates from each positive sample using PFGE or ribotyping, but less expensive screening methods, such as the allele analyses used in this study, may help address this issue.

Analysis of subtyping data revealed persistent contamination with ribotype DUP-1044A in plant C. This subtype was widespread in this plant and was isolated during each of 4 visits. Although it is difficult to establish directionality of the spread of contamination, the presence of ribotype DUP-1044A in finished products as well as in environmental sites (drains, floors) and food contact surfaces (plastic connecting tube, processing table) indicates probable postprocessing contamination of finished products from environmental sources. Jacquet et al. (1993) previously described similar contamination patterns in a cheese manufacturing plant. This group recovered $L$. monocytogenes of the same serovar and phagovar from cheese manufactured with pasteurized milk as well as from processing equipment and shelves, suggesting product contamination during the ripening process. In addition to ribotype DUP-1044A, a second ribotype, DUP-1045B, also persisted in plant $\mathrm{C}$ (Table 3 ). Our findings add to a growing body of evidence implicating persistent $L$. monocyto- 
genes subtypes in food processing plants as a major concern. Sites harboring persistent strains may facilitate the spread of $L$. monocytogenes to downstream points within the processing environment, and hence may ultimately serve as sources of finished-product contamination (Loncarevic et al., 1998; Autio et al., 1999; Miettinen et al., 1999; Norton et al., 2001; Hoffman et al., 2003; Lundén et al., 2003). Interestingly, ribotype DUP-1044A/actA type 4/hly type 1 strains had been previously associated with a 1998 multistate human listeriosis outbreak in the United States, which was linked to contaminated hot dogs and deli meats (Sauders et al., 2003). It had been postulated that persistent contamination of the processing plant environment after a construction event contributed to this outbreak. We thus hypothesize that DUP-1044A may represent a strain or clonal group that is specifically capable of persisting in food processing environments. In further support of this hypothesis, ribotype DUP-1044A also had been isolated on multiple occasions in 2 of 3 smoked-fish processing plants tested by Norton et al. (2001).

In plant A, we identified the same ribotype (DUP1062C) in a cooler room drain and, on a subsequent visit, in a crate used for transport and storage of finished products in the cooler room. This finding may indicate the crate as a vehicle of cross contamination. In this plant, ribotype DUP-1044A was isolated from both a floor and a drain sample collected during the same visit. No ribotypes persisted over more than 2 visits or at multiple sites in plant A, suggesting that persistent L. monocytogenes contamination was successfully prevented in this plant. Plant B showed the highest ribotype diversity, with a total of 5 different ribotypes isolated from this plant. Two different ribotypes persisted in different environmental sites in this plant. Ribotype DUP-1042A was isolated from crates on 3 separate visits, and DUP-1042C was isolated from drain and floor samples on 3 separate visits. These results further demonstrate the role of molecular subtyping data in environmental monitoring programs to differentiate transient from persistent contamination and to allow development of appropriate control and intervention strategies.

\section{L. monocytogenes Detection Methods}

Detection of L. monocytogenes using culture-based methods is time consuming and can be challenging, particularly if $L$. monocytogenes is present in a background of other Listeria spp. Other Listeria spp. may overgrow $L$. monocytogenes during enrichment (Beumer et al., 1996) and most selective and differential Listeria plating media do not differentiate $L$. monocytogenes from nonpathogenic Listeria spp. Thus, specific detection of $L$. monocytogenes relies on phenotypic and biochemical testing of selected Listeria-like colonies collected from plating media. Usually only a few isolates $(<5)$ are selected for screening to determine the presence of $L$. monocytogenes; samples with a high Listeria spp. to $L$. monocytogenes ratio may thus be scored incorrectly as $L$. monocytogenes negative. The increasing need for economical testing strategies with rapid turnaround times has led to the common use of Listeria spp. as an indicator for conditions that may enable the presence of $L$. monocytogenes in the food industry. Our results indicate that environmental testing for Listeria spp. and $L$. monocytogenes are likely to yield different results. For example, all 3 Latin-style fresh-cheese processing plants showed between 20 and $22 \%$ prevalence of Listeria spp. in environmental samples, which could lead to the hypothesis that similar hygiene and Listeria control programs were in place for all 3 plants. However, one plant had a significantly lower $L$. monocytogenes prevalence (4.1\%) as compared with the 2 other plants (14.5 and $15.9 \%)$. Our results thus indicate that the presence of Listeria spp. may not be a good predictor of $L$. monocytogenes contamination, and that L. monocytogenes-specific testing of environmental samples may be a more appropriate strategy for monitoring the effectiveness of $L$. monocytogenes control strategies.

Recently developed and evaluated selective and differential media for L. monocytogenes (Restaino et al., 1999) may provide a rapid and less labor-intensive alternative to Listeria spp. testing of environmental samples. LMDS represents a complete detection system, including preenrichment and enrichment broths and a differential and selective media, which can distinguish $L$. monocytogenes and L. ivanovii from other Listeria spp. based on PI-PLC production. LMCM (which is also included in LMDS) and PBBA subsequently can distinguish $L$. monocytogenes from $L$. ivanovii. We compared the performance of LMDS to the FDA methods for detection of L. monocytogenes from 246 environmental samples. The FDA method showed a sensitivity of $85.2 \%$, as compared with $48.2 \%$ for the LMDS. The LMDS preenrichment broth used in our study was an early production batch manufactured before the decision had been made to include the selective agents nalidixic acid or cycloheximide in the medium (L. Restaino, personal communication, 2000). We suspect that, as a consequence, the $L$. monocytogenes present in the samples that were falsely negative with the LMDS method may have been overgrown by other bacteria. The absence of the selective agents in the batch of media used in our study might explain why our results differ from the findings of Restaino et al. (1999), who showed that LMDS (containing a selective agent) was more sensitive 
than the USDA method for detecting L. monocytogenes from environmental samples collected in meat processing facilities. Additionally, we also evaluated the inclusion of LMPM as a third plating medium in the FDA method for detection of $L$. monocytogenes from 111 cheese samples. Listeria monocytogenes was not isolated on LMPM for 2 of 7 samples that yielded $L$. monocytogenes from the FDA-recommended plating media, Oxford and PALCAM. In contrast, in a study using the same 3 plating media to detect $L$. monocytogenes in 2000 food and environmental samples, Jinneman at al. (2003) reported that the use of LMPM, combined with Oxford or PALCAM media, allowed for improved detection of $L$. monocytogenes over the use of Oxford and PALCAM alone.

\section{CONCLUSIONS}

Whereas at least one previous listeriosis outbreak has been linked to Latin-style fresh cheese manufactured from $L$. monocytogenes-contaminated unpasteurized milk (Linnan et al., 1988), our results show that persistent environmental contamination represents another important source of finished product contamination for these products. Comprehensive environmental monitoring programs for L. monocytogenes, including specific testing for L. monocytogenes along with application of molecular subtyping strategies, are required to detect persistent contamination and to monitor the effectiveness of control strategies, which require integration of an effective sanitation program with good manufacturing practices. Whereas Listeria spp. testing is often recommended as an indicator for conditions that may enable the presence of $L$. monocytogenes, our results indicate that the presence of Listeria spp. may not be a good predictor of $L$. monocytogenes contamination. Whereas additional studies will be required to better understand correlations between Listeria positive samples and the presence of $L$. monocytogenes, the further development and refinement of rapid, inexpensive, and easy to use detection methods (e.g., chromogenic media) that allow specific detection of $L$. monocytogenes in environmental samples will clearly improve our ability to control this foodborne pathogen in the dairy industry.

\section{ACKNOWLEDGMENTS}

D. Y. Kabuki and A. Y. Kuaye were supported by FEA/UNICAMP-FAPESP-Brazil. This project was also supported by the New York State Office of Science, Technology, and Academic Research. We thank Steve Murphy and Sharinne Sukhnanand for help with sample collection and testing.

\section{REFERENCES}

Anonymous. 2001. Outbreak of listeriosis associated with homemade Mexican-style cheese-North Carolina, October 2000-January 2001. MMWR. 50:560-562.

Arimi, S. M., E. T. Ryser, T. J. Pritchard, and C. W. Donnelly. 1997. Diversity of Listeria ribotypes recovered from dairy cattle, silage, and dairy processing environments. J. Food Prot. 60:811-816.

Autio, T., S. Hielm, M. Miettinen, A. M. Sjöberg, K. Aarlisalo, J. Björkroth, and T. Mattila-Sandholm. 1999. Sources of Listeria monocytogenes contamination in a cold smoked rainbow trout processing plant detected by pulsed-field gel electrophoresis typing. Appl. Environ. Microbiol. 65:150-155.

Azadian, B. S., G. T. Finnerty, and A. D. Pearson. 1989. Cheeseborne Listeria meningitis in immunocompetent patient. Lancet 1:322-323.

Beumer, R. R., M. C. te Giffel, S. V. R. Anthonie, and L. J. Cox. 1996. The effect of acriflavin and nalidixic acid on the growth of Listeria spp. in enrichment media. Food Microbiol. 13:137-148.

Bille, J. 1990. Epidemiology of human listeriosis in Europe with special reference to the Swiss outbreak. Pages 71-74 in Foodborne Listeriosis. A. J. Miller, J. L. Smith, G. A. Somkuti, ed. Elsevier, New York, NY.

Bruce, J. 1996. Automated system rapidly identifies and characterizes microorganisms in food. Food Technol. 50:77-81.

Cox, L. J., T. Kleiss, J. L. Cordier, C. Cordellanac, P. Konkel, C. Pedrazzini, R. Beumer, and A. Siebenga. 1989. Listeria spp. in food processing, non-food, and domestic environments. Food Microbiol. 6:49-61.

Dalton, C. B., C. C. Austin, J. Sobel, P. S. Hayes, W. F. Bibb, L. M. Graves, B. Swaminathan, M. E. Proctor, and P. M. Griffin. 1997. An outbreak of gastroenteritis and fever due to Listeria monocytogenes in milk. N. Engl. J. Med. 336:100-105.

Destro, M. T., A. M. Serrano, and D. Y. Kabuki. 1991. Isolation of Listeria species from some Brazilian meat and dairy products. Food Control 2:110-112.

El Marrakchi, A., A. Hamama, and F. El Othmani. 1993. Occurrence of Listeria monocytogenes in milk and dairy products produced or imported into Morocco. J. Food Prot. 56:256-259.

Farber, J. M., and P. I. Peterkin. 1991. Listeria monocytogenes, a food-borne pathogen. Microbiol. Rev. 55:476-511.

Fleming, D. W., S. L. Cochi, K. L. Mac Donald, J. Brondum, P. S. Hayes, B. D. Plikaytis, M. B. Hoimes, A. Audurier, C. V. Broome, and A. L. Reingold. 1985. Pasteurized milk as a vehicle of infection in an outbreak of listeriosis. N. Engl. J. Med. 312:404-407.

Furlanetto, S. M., M. A. A. Santos, and C. Hara. 1996. Avaliação da eficiência de quatro meios de plaqueamento no seu isolamento. Higiene Alimentar. 10:30-34.

Genigeorgis, C., J. H. Toledo, and F. J. Garayzabal. 1991. Selected microbiological and chemical characteristics of illegally produced and marketed soft Hispanic-style cheeses in California. J. Food Prot. 54:598-601.

Gombas, D. E., Y. Chen, R. S. Clavero, and V. N. Scott. 2003. Survey of Listeria monocytogenes in ready-to-eat foods. J. Food Prot. 66:559-569.

Hitchins, A. D. 1998. Listeria monocytogenes. Pages 10-01-10-13 in Food and Drug Administration. Bacteriological Analytical Manual, 8th ed. Revision A. AOAC, Arlington, VA.

Hoffman, A. D., and M. Wiedmann. 2001. Comparative evaluation of culture and BAX polymerase chain reaction-based detection methods for Listeria spp. and Listeria monocytogenes in environmental and raw fish samples. J. Food Prot. 64:1521-1526.

Hoffman, A. D., K. L. Gall, D. M. Norton, and M. Wiedmann. 2003. Listeria monocytogenes contamination patterns for the smoked fish processing environment and for raw fish. J. Food Prot. 66:52-60.

Jacquet, C., B. Catimel, R. Brosch, C. Buchrieser, P. Dehaumont, V. Goulet, A. Lepoutre, P. Veit, and J. Rocourt. 1995. Investigations related to the epidemic strain involved in the French listeriosis outbreak in 1992. Appl. Environ. Microbiol. 61:2242-2246. 
Jacquet, C., J. Rocourt, and A. Reynaud. 1993. Study of Listeria monocytogenes contamination in a dairy plant and characterization of the strains isolated. Int. J. Food Microbiol. 20:13-22.

Jinneman, K. C., J. M. Hunt, C. A. Eklund, J. S. Wernberg, P. N. Sado, J. M. Johnson, R. S. Richter, S. T. Torres, E. Ayotte, S. J. Eliasberg, P. Istafanos, D. Bass, N. Kexel-Calabresa, W. Lin, and C. N. Barton. 2003. Evaluation and interlaboratory validation of a selective agar for phosphatidylinositol-specific phospholipase $\mathrm{C}$ activity using a chromogenic substrate to detect Listeria monocytogenes from foods. J. Food Prot. 66:441-445.

Johansson, T., L. Rantala, L. Palmu, and T. Honkanen-Buzalki. 1999. Occurrence and typing of Listeria monocytogenes strains in retail vacuum-packed fish and in a production plant. Int. J. Food Microbiol. 47:111-119.

Karpísková, R., M. Pejchalova, J. Mokrosová, J. Vytrasová, P. Smuhanová, and J. Ruprich. 2000. Application of a chromogenic medium and the PCR methods for the rapid confirmation of Listeria monocytogenes in foodstuffs. J. Microbiol. Meth. 41:267-271.

Klausner, R. B., and C. Donnelly. 1991. Environmental sources of Listeria and Yersinia in Vermont dairy plants. J. Food Prot. 54:607-611.

Krysinski, E. P., L. J. Brown, and T. J. Marchisello. 1992. Effect of cleaners and sanitizers on Listeria monocytogenes attached to product contact surfaces. J. Food Prot. 55:246-251.

Linnan, M. J., L. Mascola, X. D. Lou, V. Goulet, S. May, C. Salminen, D. W. Hird, M. L. Yonekura, P. Hayes, R. Weaver, A. Audurier, B. D. Plikaytis, S. L. Fannin, A. Kleks, and C. V. Broome. 1988. Epidemic listeriosis associated with Mexican-style cheese. N. Engl. J. Med. 319:823-828.

Loncarevic, S., E. Bannerman, J. Bille, M. L. Danielsson-Tham, and W. Tham. 1998. Characterization of Listeria strains isolated from soft and semi-soft cheeses. Food Microbiol. 15:521-525.

Lundén, J. M., T. J. Autio, A.-M. Sjöberg, and H. J. Korkeala. 2003. Persistent and nonpersistent Listeria monocytogenes contamination in meat and poultry processing plants. J. Food Prot. 66:2062-2069.

McLauchlin, J., M. H. Greenwood, and P. N. Pini. 1990. The occurrence of Listeria monocytogenes in cheese from a manufacturer associated with a case of listeriosis. Int. J. Food Microbiol. 10:255-262.

Mead, P. S., L. Slutsker, V. Deitz, L. F. McCaig, J. S. Bresee, C. Shapiro, P. M. Grifinn, and R. V. Tauxe. 1999. Food-related illness and death in the United States. Emerg. Infect. Dis. 5:607-625.

Miettinen, M. K., K. J. Björkroth, and H. J. Korkeala. 1999. Characterization of Listeria monocytogenes from an ice cream plant by serotyping and pulsed-field gel electrophoresis. Int. J. Food Microbiol. 46:187-192.

Norton, D. M., M. A. McCamey, K. J. Boor, and M. Wiedmann. 2000. Application of the BAX for screening/genus Listeria polymerase chain reaction system for monitoring Listeria species in coldsmoked fish and in the smoked fish processing environment. J. Food Prot. 63:343-346.

Norton, D. M., M. A. McCamey, K. L. Gall, J. M. Scarlett, K. J. Boor, and M. Wiedmann. 2001. Molecular studies on the ecology of
Listeria monocytogenes in the smoked fish processing industry. Appl. Environ. Microbiol. 67:198-205.

Notermans, S. H. W., J. Dufrenne, M. Leimeister-Wächter, E. Domann, and T. Chakraborty. 1991. Phosphatidylinositol-specific phospholipase $\mathrm{C}$ activity as a marker to distinguish between pathogenic and nonpathogenic Listeria species. Appl. Environ. Microbiol. 57:2666-2670.

Pritchard, T. J., K. J. Flanders, and C. W. Donnelly. 1995. Comparison of the incidence of Listeria on equipment versus environmental sites within dairy processing plants. Int. J. Food Microbiol. 26:375-384.

Restaino, L., E. W. Frampton, R. M. Irbe, G. Schabert, and H. Spitz. 1999. Isolation and detection of Listeria monocytogenes using fluorogenic and chromogenic substrates for phosphatidylinositolspecific phospholipase C. J. Food Prot. 62:244-251.

Ryser, E. T., S. M. Arimi, M. M.-C. Bunduki, and C. W. Donnelly. 1996. Recovery of different Listeria ribotypes from naturally contaminated, raw refrigerated meat and poultry products with two primary enrichment media. Appl. Environ. Microbiol. 62:17811787.

Saltijeral, J. A., V. B. Alvarez, and B. Garcia. 1999. Presence of Listeria in Mexican cheeses. J. Food Safety 19:241-247.

Sauders, B. D., E. D. Fortes, D. L. Morse, N. Dumas, J. A. Kiehlbauch, Y. Schukken, J. R. Hibbs, and M. Wiedmann. 2003. Molecular subtyping to detect human listeriosis clusters. Emerg. Infect. Dis. 9:672-680.

Silva, M. C. D., E. Hofer, and A. Tibana. 1998. Incidence of Listeria monocytogenes in cheese produced in Rio de Janeiro. Brazil. J. Food Prot. 61:354-356.

Sinde, E., and J. Carballo. 2000. Attachment of Salmonella spp. and Listeria monocytogenes to stainless steel, rubber and polytetrafluorethylene: The influence of free energy and the effect of commercial sanitizers. Food Microbiol. 17:439-447.

Sutherland, P., and R. Porritt. 1996. Dissemination and ecology of Listeria monocytogenes in Australian dairy factory environments. Food Australia 48:172,174-176,178.

Tompkin, R. B. 2002. Control of Listeria monocytogenes in the foodprocessing environment. J. Food Prot. 65:709-725.

Torres, N., and R. C. Chandan. 1981. Latin American white cheeseA review. J. Dairy Sci. 64:552-557.

USDA NASS. U.S. Department of Agriculture National Agricultural Statistics Service. 2002. Agricultural Statistics 2002, Chapter VIII. Dairy and Poultry Statistics (accessed at http://www.usda. gov/nass/pubs/agr02/acro02.htm).

Wiedmann, M., J. L. Bruce, C. Keating, A. E. Johnson, P. L. McDonough, and C. Batt. 1997. Ribotypes and virulence gene polymorphisms suggest three distinct Listeria monocytogenes lineages with differences in pathogenic potential. Infect. Immun. 65:2707-2716.

Wiedmann, M. 2002. Molecular subtyping methods for Listeria monocytogenes. J. AOAC 85:524-531.

Wong, S., D. Street, S. I. Delgado, and K. C. Klonts. 2000. Recalls of foods and cosmetics due to microbial contamination reported to the U.S. Food and Drug Administration. J. Food Prot. 63:1113-1116. 